\title{
HEMAGGLUTININS ON THE BODY SURFACE OF SEA CUCUMBER, APOSTICHOPUS JAPONICUS
}

\author{
Keisuke Ono ${ }^{1}$, Shigeyuki Tsutsui ${ }^{1}$, Tomoyuki Odaka $^{2}$, Toshiaki Miyadai ${ }^{2}$, Osamu \\ Nakamura $^{1 \S}$
}

${ }^{I}$ School of Marine Biosciences, Kitasato University, Kanagawa, Japan

${ }^{2}$ Faculty of Marine Bioscience, Fukui Prefectural University, Fukui, Japan

\begin{abstract}
The body surface is an indispensable barrier in aquatic animals for protection against diseasecausing microorganisms. However, the defense molecules on the body surface of aquatic invertebrates remain largely unexplored. To examine lectin molecules on the body surface of the Japanese sea cucumber, Apostichopus japonicus, we scraped its integument using a surgical knife. The integument extract showed agglutinating activity against rabbit red blood cells. A 15-kDa protein was successfully isolated by affinity chromatography using a fixed erythrocyte membrane and an acidic buffer as eluant. N-terminal amino acid sequencing, followed by PCR using the degenerated primer based on the N-terminal sequence and RACE PCR, were performed. A fulllength cDNA of the $15-\mathrm{kDa}$ protein contains $1438 \mathrm{bp}$, including $492 \mathrm{bp}$ of the open reading frame, which encodes 163 amino acid residues. The translated sequence is consistent with that of SJL-1, a C-type lectin, found previously in the whole-body extract of $A$. japonicus, with the exception of two amino acid residues. Reverse transcription PCR demonstrated that the gene was transcribed in the intestine, respiratory trees, and tentacles, but not in the coelomocytes and body wall, including the body surface. Western blotting, using a specific antibody raised against recombinant SJL-1, showed that the protein exists in the body surface extract and body surface rinsing, but not in the coelomic fluid. These results suggest that SJL-1 was not produced in the integument but transported to the body surface from the synthesis site through an unknown pathway.
\end{abstract}

\section{KEYWORDS}

holothurian, Apostichopus japonicus, C-type lectin, integument, SJL-1

§ Corresponding author. Tel.: +81 427789391; Fax: +81 427785010

E-mail address: naosamu@kitasato-u.ac.jp 